\title{
Catheter-Based Local Delivery of Therapeutics to the Lungs for Severe or Critically III Patients with COVID-19
}

\author{
Authors: \\ George Dangas, ${ }^{1}$ Christopher J. White, ${ }^{2}$ Nodar Kipshidze, ${ }^{3}$ \\ Jawed Fareed, ${ }^{4}$ Patrick Iversen, ${ }^{5}$ Antonio Colombo, ${ }^{6}$ \\ ${ }^{*}$ Nicholas Kipshidze ${ }^{7}$ \\ 1. Icahn School of Medicine at Mount Sinai, New York City, New York, USA \\ 2. Ochsner Clinical School, University of Queensland, Australia and Ochsner Medical \\ Center, New Orleans, Louisiana, USA \\ 3. NYU Langone Health, New York, New York, USA \\ 4. Department of Pathology and Laboratory Medicine and Department of \\ Pharmacology and Neuroscience, Cardiovascular Research Institute, Loyola \\ University Chicago, Health Sciences Division, Maywood, Illinois, USA \\ 5. USAMRID, Fort Detrick, Maryland, USA \\ 6. University Vita e Salute in San Raffaele Hospital and Columbus Hospitals, Milan, \\ Italy \\ 7. NY Cardiovascular Research, New York City, New York, USA \\ *Correspondence to NicholasO2@msn.com
}

Disclosure:

The authors have declared no conflicts of interest.

Acknowledgements:

Received:

27.04 .20

Accepted:

04.01 .21

Keywords:

Acute respiratory distress syndrome (ARDS), catheter interventions, coronavirus disease (COVID-19), local drug delivery, respiratory infections, severe acute respiratory syndrome coronavirus 2 (SARS-CoV-2).

Citation:

EMJ Innov. 2020;5[1]:46-51.

\section{INTRODUCTION}

Currently, the majority of treatment strategies reported for coronavirus disease (COVID-19) involve the systemic administration of drugs, in addition to other approaches including convalescent plasma., ${ }^{1,2}$ The pathogenesis of severe acute respiratory syndrome coronavirus 2 (SARS-CoV-2) was believed to be largely localised in the lungs. Retrospective observational studies from Wuhan, China have shown subgroups of patients with symptoms affecting the cardiovascular, renal, nervous, and digestive systems. ${ }^{3,4}$ Yet the most critical cases largely encompass those suffering from acute respiratory distress syndrome (ARDS).
Therefore, the authors propose a combination of local delivery of therapeutics directly to the lungs and adjunct systemic administration (i.e., intravenous infusion).

\section{SARS-CoV-2 Pathogenesis}

Building on initial clinical and serological reports, SARS-CoV-2 appears to mainly infect the upper and lower respiratory tract. Hoffmann et al. $^{5}$ demonstrated that the virus requires two host cell factors for successful viral entry: angiotensin-converting enzyme 2 (ACE-2) and transmembrane proteases, serine 2 (TMPRSS2). Previous studies have indicated the expression of both factors in human lung tissue.6,7 Wang et al. ${ }^{8}$ analysed specimens taken from the lower and 
upper respiratory tract, faeces, blood, and urine samples of laboratory-confirmed COVID-19 cases, in which a majority of live virus was obtained from the lower respiratory tract in bronchoalveolar lavage fluid (93\%). Therefore, if viral replication is primarily occurring in the lungs, a localised therapeutic approach could prove to be just as, if not more effective than, a systemic approach. Similarly, proinflammatory cytokines (i.e., IL-6 or TNF-a) activated by a viral infection in the lungs will proliferate mainly in the lungs. Radiological studies have demonstrated bilateral interstitial lung inflammation and fibrosis, characterised by ground glass opacity, crazy-paving pattern, and consolidation in lung CT scans. ${ }^{9}$ Drugs candidates that target these proinflammatory cytokines (IL2, IL-6, TMPRSS2) would again benefit from a localised delivery to counteract the damaging effects of ARDS. Considering that COVID-19 primarily affects the lungs, the authors propose a transcatheter approach to locally deliver pharmaceutical therapies (Table 1) as an adjunct to systemic therapy.

Table 1: Drug candidates for local delivery in the treatment of patients with coronavirus disease (COVID-19).

\begin{tabular}{|c|c|c|}
\hline Mode of Action & Class & Drugs \\
\hline \multirow[t]{4}{*}{ Antiviral } & Nucleotide prodrug & Remdesivir \\
\hline & Protein & $\begin{array}{l}\text { Human recombinant soluble ACE2 } \\
\text { (hrsACE2) }\end{array}$ \\
\hline & Photosensitiser & LS11 \\
\hline & Polysaccharide & Pentosan polysulfate \\
\hline \multirow[t]{4}{*}{ Anti-inflammatory } & Immune modulator & Colchicine \\
\hline & \multirow[t]{2}{*}{ Glycosaminoglycan } & Low-molecular-weight heparin \\
\hline & & Unfractionated heparin \\
\hline & mTOR inhibitor & Sirolimus \\
\hline \multirow[t]{3}{*}{ Antithrombotic } & \multirow[t]{2}{*}{ Glycosaminoglycan } & Danaparoid \\
\hline & & Sulodexide \\
\hline & Synthetic oligosaccharide & Fondaparinux \\
\hline Bradykinin storm inhibitor & Immune modulator & Icatibant \\
\hline \multirow[t]{8}{*}{ Cytokine storm inhibitor } & \multirow[t]{5}{*}{ Monoclonal antibody } & Sarilumab \\
\hline & & Tocilizumab \\
\hline & & Baricitinib \\
\hline & & Risankizumab \\
\hline & & Lenzilumab \\
\hline & \multirow[t]{2}{*}{ Hormone } & Prednisolone \\
\hline & & Dexamethasone \\
\hline & Interferons & Interferon $\beta-1 a$ \\
\hline \multirow{2}{*}{$\begin{array}{l}\text { Endothelial protectant and } \\
\text { vasculoprotective }\end{array}$} & Nucleic acid-based drug & Defibrotide \\
\hline & Oestrogen & 17ß-oestradiol \\
\hline \multirow[t]{3}{*}{ Thrombolytic } & Protein & Tissue plasminogen activator \\
\hline & Plasminogen activator & Urokinase \\
\hline & Enzyme & Streptokinase \\
\hline ADP receptor inhibitor & Antiplatelet drug & Cangrelor \\
\hline
\end{tabular}




\section{RATIONALE FOR LOCAL THERAPEUTICS}

\section{Drug Delivery Methods}

There are numerous drug candidates being investigated for COVID-19 treatment that either target the virus lifecycle (entry, replication, or exocytosis) or modulate the host immune response (stimulate viral interferons or inhibit cytokine storm). ${ }^{10}$ Regardless of the candidate, these therapeutics can be administered as a tablet, intravenous infusion, or subcutaneous injection. Orally administered drugs may be affected by first-pass metabolism, in which a majority of the therapeutic is metabolised by the liver. Once it passes the liver, the bioavailability of the drug may be greatly reduced at which point the drug circulates systemically. Intravenous administration typically overcomes issues regarding bioavailability, though the drug again circulates systemically once it passes the lungs. A localised delivery of therapeutics (Figure 1) could increase the bioavailability at the site of action (i.e., the lungs).

\section{LOCAL DRUG DELIVERY APPROACHES}

\section{Pulmonary Artery Delivery}

One possible solution is to administer drugs locally using a Swan-Ganz catheter and administering the drug through the pulmonary artery. This could be performed bedside. Some intensive care units or COVID-19 treatment floors may already have catheterised their patients with triple-lumen internal jugular sheaths, in which case a Swan-Ganz approach would be easy. Two large, multicentre, randomised controlled studies looked at the use of pulmonary artery catheters (PAC) in patients with shock and ARDS.11,12

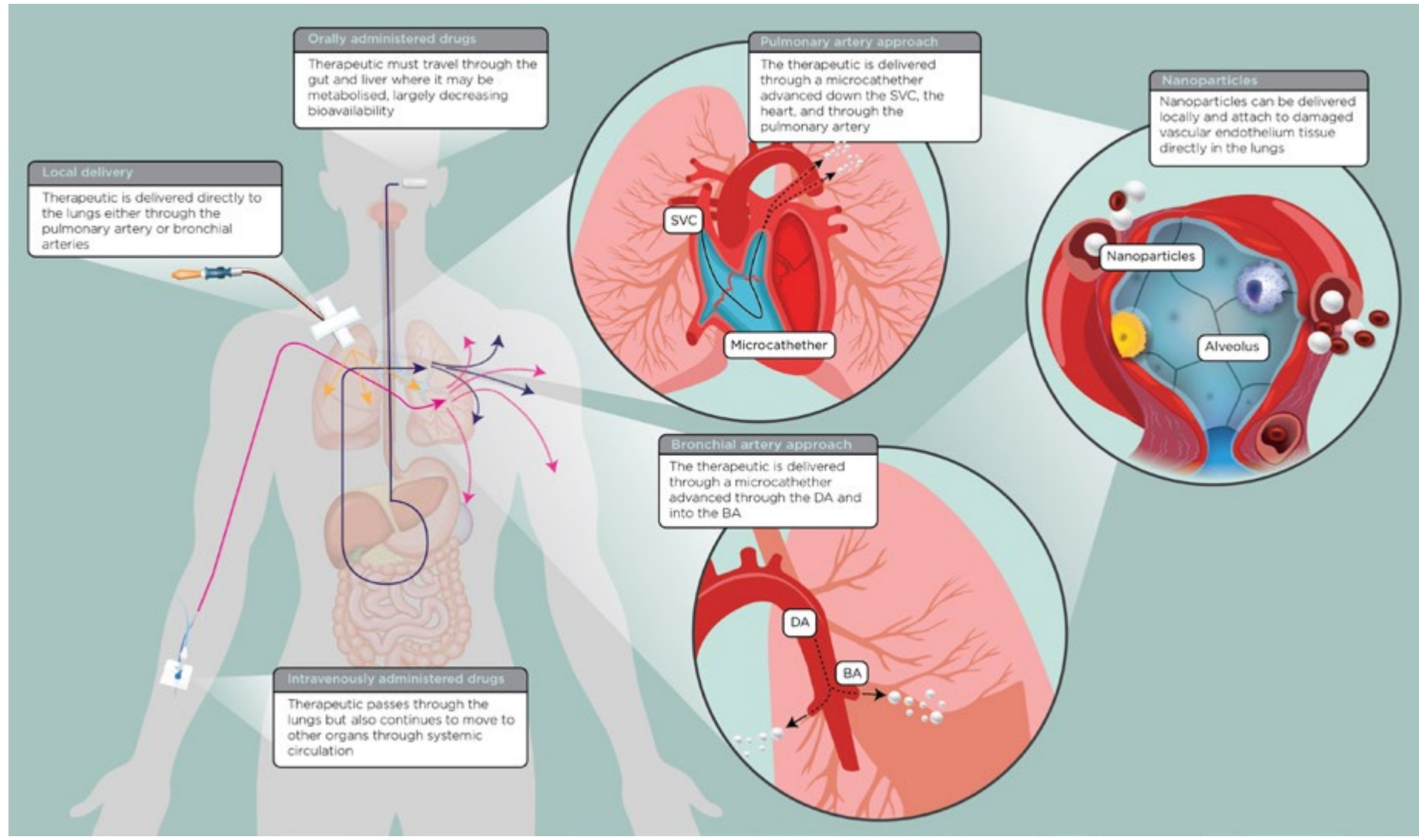

Figure 1: A simplified overview of orally administered (i.e., pill or oral suspension), intravenously administered, and locally delivered drugs for patients with coronavirus disease (COVID-19).

Blowout panels describe two local delivery approaches: the pulmonary artery and BA. The third panel describes the use of nanoparticles, which can be used with any local delivery approach.

BA: bronchial arteries; DA: descending aorta; SVC: superior vena cava. 
PAC in the context of these trials were used to measure ventricular filling pressures, cardiac output, and other haemodynamic data, which the clinicians then used to guide treatment decisions. While there was no significant benefit shown in the use of PAC to guide decisionmaking, there was no difference in mortality between the groups who did and did not receive a PAC. This may indicate that the use of PAC in patients with ARDS or acute lung injury does not pose an increased risk for mortality. Both studies indicated PAC-associated arrythmias as the most common complication. In the French PAC study, additional complications included arterial puncture and haemothorax. No deaths were attributable to ventricular fibrillation or arrhythmia. Several patients developed positive bacterial cultures following PAC insertion. ${ }^{12}$ In the National Heart, Lung, and Blood Institute (NHLBI) study, the rate of complications was no different between groups, and no deaths were related to the insertion of a catheter. ${ }^{11}$ In the setting of COVID-19, the risk of PAC-acquired bacterial infection could be low because most hospitalised patients requiring central catheter insertion are being given antibiotics prophylactically to protect against secondary infections. ${ }^{13,14}$ Local delivery of antibiotics in the form of aerosol inhalations are approved for two antibiotics in the USA (aminoglycoside and monobactam), with numerous ongoing clinical trials assessing the efficacy of aerosolised antibiotics or antibiotics reformulated as nanoparticles for chronic pulmonary infections or cystic fibrosis. ${ }^{15,16}$ In COVID-19, the drugs that target proinflammatory cytokines common in ARDS can be administered locally and several times if needed.

\section{Transarterial Local Delivery}

Transarterial local delivery is a well-established procedure, including local lung therapy via the bronchial arteries using microcatheters. ${ }^{17-19}$ With a localised therapeutic approach for COVID-19, the treating physician could administer drugs through the bronchial artery. However, given the complexity, a transarterial approach would require the clinician to perform the intervention using imaging. Yet, administering therapeutics through the bronchial artery may provide the greatest increase in bioavailability in comparison to other methods.

\section{CATHETER-DIRECTED THROMBOLYSIS}

There is growing evidence of viral coagulopathy developing in a subgroup of patients with COVID-19. Viral coagulopathy is not uncommon in other respiratory viruses, including infection from SARS-CoV-1 and 2009 pandemic H1N1 ( $\mathrm{pH} 1 \mathrm{~N} 1)$. Patients affected by SARS or $\mathrm{pH} 1 \mathrm{~N} 1$ developed intravascular thrombi, microthrombi, intra-alveolar haemorrhage, fibrin deposition in the lungs, and diffuse alveolar damage..$^{20-22}$ Several clinicopathologic reports on COVID-19 have reported postmortem findings of venous thromboembolism and thrombosis of small and midsized pulmonary arteries, as well as diffuse alveolar damage, oedema, hyaline membranes, and proliferation of pneumocytes and fibroblasts. ${ }^{23-25}$ Catheter-directed thrombolysis (CDT) could prove to be another powerful therapeutic to treat COVID-19-associated pulmonary embolism. CDT can be performed using a PAC positioned in the pulmonary artery proximal to the location of the thrombus or thrombi. Following successful positioning, a thrombolytic agent is infused through the positioned PAC, such as tissue plasminogen activator, streptokinase, or urokinase. ${ }^{26}$ CDT could reverse the hypercoagulable condition demonstrated in COVID-19 ARDS, resulting in improvements in the cardiovascular and pulmonary function. Systemic thrombolysis in the context of therapy to treat pulmonary embolism is associated with an increased risk of adverse effects, including major bleeding and stroke. ${ }^{27}$ Two previous trials assessing the efficacy of CDT versus systemic thrombolysis demonstrated improved cardiovascular haemodynamics following CDT administration of tissue plasminogen activator, with no increased risk of intracranial haemorrhaging in CDT.28,29 Moreover some investigators support catheter-directed treatment as a potential first-line therapeutic approach for Covid-19. ${ }^{30}$ However, large studies are warranted to determine the optimal dosing regimen of systemic thrombolysis and CDT with or without therapeutic anticoagulation in COVID-19 ARDS .

\section{Targeted Delivery Using Microparticles}

Local therapeutic administration can be buttressed by using different types of drug delivery vehicles (nanoparticle drug carriers, 
liposomes, viral vectors, or microbubbles). The latter adhere to sites of damaged vascular endothelium and thus may be a method of systemically targeting delivery of therapeutics to lungs damaged in SARS-CoV-2 infection. For example, perfluorobutane gas microbubbles with a coating of dextrose and albumin efficiently bind to different pharmaceuticals. These 0.3-10.0 $\mu \mathrm{m}$ particles bind to sites of vascular injury. ${ }^{31}$ Further, the perfluorobutane gas is an effective cell membrane fluidiser. The potential advantages of microbubble carrier delivery include none to minimal (additional) vessel injury through delivery, no resident polymer to degrade and lead to eventual inflammation, rapid bolus delivery, and repeated delivery. Microbubble carriers were successfully used in different animal models and clinical trials to deliver antisense oligonucleotide and/or sirolimus to the injured vascular bed. 32,33

\section{LIMITATIONS}

There are several logistical and clinical challenges one must consider when planning a local therapeutic approach. During the severe and/or critical period or period of illness, patients with
COVID-19 are highly contagious, at which time the virus may spread via droplets or become aerosolised during intubation or other aerosolproducing conditions. Therefore, the physical movement and intervention of these patients in catheterisation laboratories need to be highly controlled to minimise the risk of transmitting disease to uninfected patients, healthcare providers, or hospital staff. However, with growing evidence for significant damage to the cardiovascular system including heart failure, stent thrombosis, and acceleration of atherosclerosis of coronary arteries, percutaneous coronary interventions are unavoidable and warranted. Sensitivity to contrast media in these patients is not documented and the use of contrast should be approached with caution.

\section{CONCLUSIONS}

Despite the possible challenges, local drug delivery could prove to be a powerful tool in treating patients with severe or critical cases of COVID-19. Feasibility studies will help to determine the safety and preliminary efficacy of this approach.

\section{References}

1. Wang $D$ et al. Clinical characteristics of 138 hospitalized patients with 2019 novel coronavirus-infected pneumonia in Wuhan, China. JAMA. 2020;323(11):1061-9.

2. Young BE et al. Epidemiologic features and clinical course of patients infected with SARS-CoV-2 in Singapore. JAMA. 2020;323(15):148894.

3. Han C et al. Digestive symptoms in COVID-19 patients with mild disease severity: clinical presentation, stool viral RNA testing, and outcomes. Am J Gastroenterol. 2020;115(6):916-23.

4. Mao L et al. Neurologic manifestations of hospitalized patients with coronavirus disease 2019 in Wuhan, China. JAMA Neurol. 2020;77(6):683-90.

5. Hoffmann M et al. SARS-CoV-2 cell entry depends on ACE2 and TMPRSS2 and is blocked by a clinically proven protease inhibitor. Cell. 2020;181(2):271-80.e8.

6. Hamming I et al. Tissue distribution of ACE2 protein, the functional receptor for SARS coronavirus. A first step in understanding SARS pathogenesis. J
Pathol. 2004;203(2):631-7.

7. Vaarala $\mathrm{MH}$ et al. Expression of transmembrane serine protease TMPRSS2 in mouse and human tissues. J Pathol. 2001;193(1):134-40.

8. Wang $W$ et al. Detection of SARSCoV-2 in different types of clinical specimens. JAMA. 2020;323(18):18434.

9. Shi $\mathrm{H}$ et al. Radiological findings from 81 patients with COVID-19 pneumonia in Wuhan, China: a descriptive study. Lancet Infect Dis. 2020;20(4):425-34.

10. Sanders JM et al. Pharmacologic treatments for coronavirus disease 2019 (COVID-19): a review. JAMA. 2020;323(18):1824-36.

11. National Heart, Lung, and Blood Institute Acute Respiratory Distress Syndrome (ARDS) Clinical Trials Network; Wheeler AP et al. Pulmonary-artery versus central venous catheter to guide treatment of acute lung injury. N Engl J Med. 2006;354(21):2213-24

12. Richard C et al. Early use of the pulmonary artery catheter and outcomes in patients with shock and acute respiratory distress syndrome: a randomized controlled trial. JAMA 2003;290(20):2713-20.

13. Zhou F et al. Clinical course and risk factors for mortality of adult inpatients with COVID-19 in Wuhan, China: a retrospective cohort study. Lancet. 2020;395(10229):1054-62.

14. Cummings MJ et al. Epidemiology, clinical course, and outcomes of critically ill adults with COVID-19 in New York City: a prospective cohort study. Lancet. 2020;395(10239):176370 .

15. Moreno-Sastre M et al. Pulmonary drug delivery: a review on nanocarriers for antibacterial chemotherapy. J Antimicrob Chemother. 2015;70(11):2945-55.

16. Park $\mathrm{C}-\mathrm{W}$ et al. Pulmonary inhalation aerosols for targeted antibiotics drug delivery. Eur Pharm Rev. 2011;1.

17. Hamahata A et al. Direct delivery of low-dose 7-nitroindazole into the bronchial artery attenuates pulmonary pathophysiology after smoke inhalation and burn injury in an ovine model. Shock. 2011;36(6):575-9.

18. Shao $\mathrm{H}$ et al. Bronchial artery embolization for hemoptysis: a 
retrospective observational study of 344 patients. Chin Med J (Engl). 2015;128(1):58-62.

19. Panda A et al. Bronchial artery embolization in hemoptysis: a systematic review. Diagn Interv Radiol. 2017;23(4):307-17.

20. Hwang DM et al. Pulmonary pathology of severe acute respiratory syndrome in Toronto. Mod Pathol. 2005;18(1):1-10.

21. Mauad T et al. Lung pathology in fatal novel human influenza A (H1N1) infection. Am J Respir Crit Care Med. 2010;181(1):72-9

22. Bunce PE et al. Pandemic H1N1 influenza infection and vascular thrombosis. Clin Infect Dis. 2011:52(2):e14-7.

23. Lax SF et al. Pulmonary arterial thrombosis in COVID-19 with fatal outcome: results from a prospective, single-center, clinicopathologic case series. Ann Intern Med. 2020:DOI:10.7326/M20-2566.

24. Schaller $T$ et al. Postmortem examination of patients with COVID-19. JAMA. 2020;323(24):2518 20.

25. Ackermann $\mathrm{M}$ et al. Pulmonary vascular endothelialitis, thrombosis, and angiogenesis in Covid-19. N Engl J Med. 2020;383(2):120-8.

26. Konstantinides SV et al. 2019 ESC Guidelines for the diagnosis and management of acute pulmonary embolism developed in collaboration with the European Respiratory Society (ERS): the task force for the diagnosis and management of acute pulmonary embolism of the European Society of Cardiology (ESC). Eur Respir J. 2019;54(3):1901647.

27. Schultz J et al. Catheter-based therapies in acute pulmonary embolism. Eurolntervention. 2018;13(14):1721-7.

28. Pei DT et al. Meta-analysis of catheter directed ultrasound-assisted thrombolysis in pulmonary embolism. Am J Cardiol. 2019;124(9):1470-7.

29. Hennemeyer $C$ et al. Outcomes of catheter-directed therapy plus anticoagulation versus anticoagulation alone for submassive and massive pulmonary embolism. Am J Med. 2019;132(2):240-6.

30. Roncon $L$ et al. Fibrinolysis in COVID-19 patients with hemodynamic unstable acute pulmonary embolism: yes or no? J Thromb Thrombolysis. 2020;50(1):221-2.

31. Porter TR et al. Targeted vascular delivery of antisense molecules using intravenous microbubbles. Cardiovasc Revasc Med. 2006;7(1):25-33.

32. Kipshidze NN et al. Novel site-specific systemic delivery of Rapamycin with perfluorobutane gas microbubble carrier reduced neointimal formation in a porcine coronary restenosis model. Catheter Cardiovasc Interv. 2005:64(3):389-94

33. Porter TR et al. Clinical applications of ultrasonic enhancing agents in echocardiography: 2018 American Society of Echocardiography Guidelines update. J Am Soc Echocardiogr. 2018;31(3):241-74. 